\title{
Common fixed point results of Ciric-Suzuki-type inequality for multivalued maps in compact metric spaces
}

\author{
Fawzia Shaddad $^{1 *}$, Mohd Salmi Md Noorani ${ }^{1}$ and Saud M Alsulami ${ }^{2}$
}

"Correspondence:

fzsh99@gmail.com

'School of Mathematical Sciences,

Faculty of Science and Technology,

Universiti Kebangsaan Malaysia,

UKM, Bangi, Selangor Darul Ehsan

43600, Malaysia

Full list of author information is

available at the end of the article

\begin{abstract}
The aim of this paper is to introduce a new type of multivalued operators similar to those of Ciric-Suzuki type. A common fixed point theorem for multivalued maps on metric spaces satisfying Ciric-Suzuki-type inequality is proved. Applications to certain functional equations arising in dynamic programming are also discussed.
\end{abstract}

MSC: 54E50; 54H25; 45G05

Keywords: metric space; common fixed point; Urysohn integral equations

\section{Introduction and preliminaries}

In 1937, Von Neumann [1] initiated fixed point theory for multivalued mappings in the study of game theory. Indeed, the fixed point theorems for multivalued mappings are quite useful in control theory and have been frequently used in solving many problems of economics and game theory. Successively, Nadler [2] initiated the development of the geometric fixed point theory for multivalued mappings. He used the concept of the Hausdorff metric to establish the multivalued contraction principle containing the Banach contraction principle as a special case.

Consistent with Nadler ([3], p.620), $(X, d)$ and $C l(X)$ will denote a metric space and the collection of all nonempty closed subsets of $X$, respectively. For $A, B \in C l(X)$ and $\epsilon>0$,

$$
\begin{aligned}
& N(\epsilon, A)=\{x \in X: d(x, a)<\epsilon \text { for some } a \in A\}, \\
& E_{A, B}=\{\epsilon>0: A \subseteq N(\epsilon, B), B \subseteq N(\epsilon, A)\}, \\
& H(A, B)= \begin{cases}\inf E_{A, B} & \text { if } E_{A, B} \neq \emptyset, \\
+\infty & \text { if } E_{A, B}=\emptyset .\end{cases}
\end{aligned}
$$

The hyperspace $(\mathrm{Cl}(X), H)$ is called the generalized Hausdorff metric space induced by the metric $d$ on $X$.

Later, Doric and Lazovic [4] have extended and generalized fixed point theorems of Ciric [5], Kikkawa and Suzuki [6], Nadler [2], and others as follows.

Theorem 1.1 [4] Define a nonincreasing function $\varphi$ from $[0,1)$ into $(0,1]$ by

$$
\varphi(r)= \begin{cases}1 & \text { if } 0 \leq r<\frac{1}{2}, \\ 1-r & \text { if } \frac{1}{2} \leq r<1 .\end{cases}
$$

O2014 Shaddad et al.; licensee Springer. This is an Open Access article distributed under the terms of the Creative Commons Attribution License (http://creativecommons.org/licenses/by/2.0), which permits unrestricted use, distribution, and reproduction in any medium, provided the original work is properly cited. 
Let $(X, d)$ be a complete metric space and $T: X \rightarrow C B(X)$. Assume that there exists $r \in[0,1)$ such that for every $x, y \in X$,

$$
\varphi(r) d(x, T x) \leq d(x, y) \quad \text { implies } \quad H(T x, T y) \leq r M(x, y),
$$

where

$$
M(x, y)=\max \left\{d(x, y), d(x, T x), d(y, T y), \frac{1}{2}(d(x, T y)+d(y, T x))\right\} .
$$

Then there exists $v \in X$ such that $v \in T v$.

On the other hand, Suzuki [7] introduced the following theorem. This result involves a new type of contractive mapping and hence generalizes the well-known Edelstein fixed point theorem in [8].

Theorem 1.2 [7] Let $(X, d)$ be a compact metric space, and letf be a mapping on X. Assume that

$$
\frac{1}{2} d(x, f x)<d(x, y) \quad \text { implies } \quad d(f x, f y)<d(x, y)
$$

for $x, y \in X$. Then $f$ has a unique fixed point.

Many authors have proved numerous fixed point theorems as generalization of Nadler's theorem (see [9-12]).

In 2011, Haghi et al. [13] gave a very useful lemma which we need in our work.

Lemma 1.3 [13] Let $X$ be a nonempty set and $f: X \rightarrow X$ be a function. Then there exists a subset $E \subseteq X$ such that $f(E)=f(X)$ and $f: E \rightarrow X$ is one-to-one.

In this manuscript, the well-known results of Suzuki [7], Edelstein [8] and Doric and Lazovic [4] have been merged to complement a multitude of related results from the literature. Moreover, we use Lemma 1.3 to obtain a common fixed point theorem for multivalued maps on a metric space. Finally, as an application, the existence of common solutions of certain functional equations arising in dynamic programming is proved.

\section{Fixed point results}

Theorem 2.1 Let $(X, d)$ be a compact metric space, and let $T: X \rightarrow C l(X)$. Assume that there exists $\alpha \in\left[0, \frac{1}{2}\right)$ such that

$$
\frac{1}{2} d(x, T x)<d(x, y) \quad \text { implies } \quad H(T x, T y)<\alpha M(x, y),
$$

where

$$
M(x, y)=\max \left\{d(x, y), d(x, T x), d(y, T y), \frac{1}{2}(d(x, T y)+d(y, T x))\right\}
$$

for all $x, y \in X$. Then $T$ has a fixed point. 
Proof Let $\left\{x_{n}\right\}$ be a sequence in $X$. We have that $T x_{n}$ is a closed set, so we can find a sequence $\left\{z_{n}\right\}$ in $X$ such that $z_{n} \in T x_{n}$ and $d\left(x_{n}, z_{n}\right)=d\left(x_{n}, T x_{n}\right)$. Now, put $\beta=\inf \{d(x, T x)$ : $\left.x \in X\}=\inf _{\left\{\inf _{z \in T x}\right.} d(x, z): x \in X\right\} . X$ is compact, without loss of generality, we may assume that the sequences $\left\{x_{n}\right\}$ and $\left\{z_{n}\right\}$ converge to $v$ and $w$ in $X$, respectively. Let

$$
\lim d\left(x_{n}, z_{n}\right)=\lim d\left(x_{n}, T x_{n}\right)=\lim d\left(x_{n}, w\right)=d(v, w)=\beta
$$

We want to show that $\beta=0$. Suppose not, that is, $\beta>0$. Thus, we can choose $n_{0} \in \mathbb{N}$ such that

$$
d\left(x_{n}, T x_{n}\right)<\frac{3}{2} \beta \quad \text { and } \quad \frac{3}{4} \beta<d\left(x_{n}, w\right)
$$

for $n \in \mathbb{N}$ with $n \geq n_{0}$. Thus $\frac{1}{2} d\left(x_{n}, T x_{n}\right)<d\left(x_{n}, w\right)$ for $n \geq n_{0}$. This implies

$$
H\left(T x_{n}, T w\right)<\alpha M\left(x_{n}, w\right)
$$

where

$$
M\left(x_{n}, w\right)=\max \left\{d\left(x_{n}, w\right), d\left(x_{n}, T x_{n}\right), d(w, T w), \frac{1}{2}\left(d\left(x_{n}, T w\right)+d\left(w, T x_{n}\right)\right)\right\} .
$$

Since $z_{n} \in T x_{n}, d\left(z_{n}, T w\right) \leq H\left(T x_{n}, T w\right)$. Thus

$$
d\left(z_{n}, T w\right)<\alpha M\left(x_{n}, w\right) .
$$

Moreover, $d\left(w, T x_{n}\right) \leq d\left(w, z_{n}\right)$. So

$$
M\left(x_{n}, w\right) \leq \max \left\{d\left(x_{n}, w\right), d\left(x_{n}, T x_{n}\right), d(w, T w), \frac{1}{2}\left(d\left(x_{n}, z_{n}\right)+d\left(z_{n}, T w\right)+d\left(w, z_{n}\right)\right)\right\}
$$

Let $n$ attend to $\infty$, then we have

$$
d(w, T w) \leq \alpha M(v, w)
$$

where

$$
\begin{aligned}
M(v, w) & \leq \max \left\{d(v, w), d(v, w), d(w, T w), \frac{1}{2}(d(v, w)+d(w, T w)+d(w, w))\right\} \\
& =\max \left\{d(v, w), d(w, T w), \frac{1}{2}(d(v, w)+d(w, T w))\right\}
\end{aligned}
$$

but $\frac{1}{2}(d(v, w)+d(w, T w)) \leq \max \{d(v, w), d(w, T w)\}$. Thus, $M(v, w) \leq \max \{d(v, w), d(w, T w)\}$. From (2.1), we have

$$
d(w, T w) \leq \alpha \max \{d(v, w), d(w, T w)\} \leq \alpha d(v, w)=\alpha \beta<\beta
$$

which is a contradiction to the definition of $\beta$. Hence, $\beta=0$. 
We want to prove that $T$ has a fixed point. Assume, on the contrary, that $x_{n} \notin T x_{n}$ for any $n \in \mathbb{N}$. We have $z_{n} \rightarrow w$ and $w=v$ thus $z_{n} \rightarrow v$, then $v \in T x_{n}$.

First, we want to show that

$$
d(v, T u) \leq \alpha \max \{d(v, u), d(u, T u)\} \quad \text { for every } u \in X \backslash\{v\} .
$$

We have $x_{n} \rightarrow v$ and $z_{n} \rightarrow v$, that is, there exist $n_{1}, n_{2} \in \mathbb{N}$ such that

$$
d\left(v, x_{n}\right) \leq \frac{1}{3} d(v, u) \quad \text { and } \quad d\left(v, z_{n}\right) \leq \frac{1}{3} d(v, u) \quad \text { for every } n>N=\max \left\{n_{1}, n_{2}\right\}
$$

so we have, for any $n>N$,

$$
\begin{aligned}
d\left(x_{n}, T x_{n}\right) & \leq d\left(x_{n}, z_{n}\right) \\
& \leq d\left(x_{n}, v\right)+d\left(v, z_{n}\right) \\
& \leq \frac{2}{3} d(v, u) \\
& =d(v, u)-\frac{1}{3} d(v, u) \\
& \leq d(v, u)-d\left(v, x_{n}\right) \\
& \leq d\left(x_{n}, u\right) .
\end{aligned}
$$

Thus $\frac{1}{2} d\left(x_{n}, T x_{n}\right)<d\left(x_{n}, u\right)$, which implies $H\left(T x_{n}, T u\right)<\alpha M\left(x_{n}, u\right)$, where

$$
M\left(x_{n}, u\right)=\max \left\{d\left(x_{n}, u\right), d\left(x_{n}, T x_{n}\right), d(u, T u), \frac{1}{2}\left(d\left(u, T x_{n}\right)+d\left(x_{n}, T u\right)\right)\right\}
$$

but $v \in T x_{n}$, thus

$$
d(v, T u)<\alpha \max \left\{d\left(x_{n}, u\right), d\left(x_{n}, T x_{n}\right), d(u, T u), \frac{1}{2}\left(d\left(u, T x_{n}\right)+d\left(x_{n}, T u\right)\right)\right\} .
$$

We have $d\left(u, T x_{n}\right)+d\left(x_{n}, T u\right) \leq d(u, v)+d\left(x_{n}, T u\right)$. Then (2.3) becomes

$$
d(v, T u)<\alpha \max \left\{d\left(x_{n}, u\right), d\left(x_{n}, T x_{n}\right), d(u, T u), \frac{1}{2}\left(d(u, v)+d\left(x_{n}, T u\right)\right)\right\} .
$$

By taking $n \rightarrow \infty$, we obtain

$$
d(v, T u) \leq \alpha \max \left\{d(v, u), d(u, T u), \frac{1}{2}(d(u, v)+d(v, T u))\right\} .
$$

If $d(u, v) \leq d(v, T u)$, then

$$
\begin{aligned}
d(v, T u) & \leq \alpha \max \{d(v, u), d(u, T u), d(v, T u)\} \\
& \leq \alpha \max \{d(v, u), d(u, T u)\}
\end{aligned}
$$

and if $d(v, T u) \leq d(u, v)$, then $d(v, T u) \leq \alpha \max \{d(v, u), d(u, T u)\}$. Thus, for any $u \in X \backslash\{v\}$,

$$
d(v, T u) \leq \alpha \max \{d(v, u), d(u, T u)\} .
$$


Second, we want to prove that for every $x \in X$,

$$
H(T x, T v)<\alpha \max \left\{d(x, v), d(v, T v), d(x, T x), \frac{1}{2}(d(x, T v)+d(v, T x))\right\} .
$$

If $x=v$, it is trivial. Let $x \neq v$. Then, for every $n \in \mathbb{N}$, there exists a sequence $y_{n} \in T x$ such that $d\left(v, y_{n}\right) \leq d(v, T x)+\frac{1}{n} d(v, x)$. For all $n \in \mathbb{N}$, by using (2.2) we have

$$
\begin{aligned}
d(x, T x) & \leq d\left(x, y_{n}\right) \\
& \leq d(x, v)+d\left(v, y_{n}\right) \\
& \leq d(x, v)+d(v, T x)+\frac{1}{n} d(v, x) \\
& <d(x, v)+\alpha \max \{d(v, x), d(x, T x)\}+\frac{1}{n} d(v, x) .
\end{aligned}
$$

If $d(x, T x) \leq d(v, x)$, then $d(x, T x) \leq\left(1+\alpha+\frac{1}{n}\right) d(v, x)$. When $n \rightarrow \infty$, we have

$$
d(x, T x) \leq(1+\alpha) d(v, x)<2 d(v, x) .
$$

Hence, we find that $\frac{1}{2} d(x, T x)<d(v, x)$.

If $d(v, x) \leq d(x, T x)$, then $d(x, T x) \leq d(x, v)+\alpha d(x, T x)+\frac{1}{n} d(v, x)$. Thus,

$$
(1-\alpha) d(x, T x)<\left(1+\frac{1}{n}\right) d(v, x)
$$

gives when $n \rightarrow \infty$

$$
(1-\alpha) d(x, T x) \leq d(v, x)
$$

Since $\frac{1}{2}<1-\alpha$, therefore $\frac{1}{2} d(x, T x)<d(v, x)$. Now, for every $x \in X \backslash\{v\}$, we have

$$
\frac{1}{2} d(x, T x)<d(v, x)
$$

which implies (2.4).

Finally, from (2.4) for any $n>N$, we get

$$
H\left(T x_{n}, T v\right)<\alpha \max \left\{d\left(x_{n}, v\right), d(v, T v), d\left(x_{n}, T x_{n}\right), \frac{1}{2}\left(d\left(x_{n}, T v\right)+d\left(v, T x_{n}\right)\right)\right\} .
$$

We have $z_{n} \in T x_{n}$, thus $d\left(z_{n}, T v\right) \leq H\left(T x_{n}, T v\right)$. If $n \rightarrow \infty$, then we obtain

$$
d(v, T v) \leq \alpha d(v, T v)
$$

As $\alpha<\frac{1}{2}$, we obtain that $d(v, T v)=0$. Since $T v$ is closed, thus we reach the conclusion $v \in T v$. This contradicts the assumption that $T$ has no fixed point. Hence, $T$ has a fixed point. 
Example 2.2 Let $X=[0,1]$ be endowed with the usual metric and $T: X \rightarrow C l(X)$ be defined by

$$
T x= \begin{cases}\left\{\frac{x}{6}\right\} & \text { if } x \in[0,1), \\ \left\{0, \frac{1}{6}\right\} & \text { if } x=1,\end{cases}
$$

with $\alpha=\frac{1}{4}$. Then $T$ satisfies the assumptions in Theorem 2.1.

Proof If $x=y$, it is trivial. For $x \neq y$ we have the following:

(1) $x, y \in[0,1)$ then

$$
H(T x, T y)=\left|\frac{x-y}{6}\right|<\frac{1}{4}|x-y|=\alpha d(x, y) \leq \alpha M(x, y) ;
$$

(2) $x \in[0,1)$ and $y=1$ or $(x=1$ and $y \in[0,1))$

$$
H(T x, T y) \leq \frac{1}{6}<\frac{1}{4}\left(\frac{5}{6}\right)=\alpha d(1, T 1)=\alpha d(y, T y) \leq \alpha M(x, y) .
$$

Hence, the assumptions of Theorem 2.1 are satisfied and $\{0\}$ is a fixed point.

\section{Common fixed point results}

Theorem 3.1 Let $(X, d)$ be a metric space, $Y$ be a nonempty set, $g: Y \rightarrow X$ and $T: Y \rightarrow$ $C l(X)$ such that $T(Y) \subseteq g(Y)$ and $g(Y)$ is a compact subspace of $X$. Assume that for $x, y \in Y$ there exists $\alpha \in\left[0, \frac{1}{2}\right)$ such that

$$
\frac{1}{2} d(g x, T x)<d(g x, g y) \quad \text { implies } \quad H(T x, T y)<\alpha M(g x, g y)
$$

where

$$
M(g x, g y)=\max \left\{d(g x, g y), d(g x, T x), d(g y, T y), \frac{1}{2}(d(g x, T y)+d(g y, T x))\right\}
$$

and $g x=$ gy implies $T x=T y$. Then $T$ and $g$ have a coincidence point, that is, there exists $v \in Y$ such that $g v \in T v$. If $Y=X$, then $g$, $T$ have a common fixed point provided that $g T v \subset \operatorname{Tg} v$ at $v$.

Proof By Lemma 1.3, there exists $E \subseteq Y$ such that $g(E)=g(Y)$ and $g: E \rightarrow X$ is one-to-one. We define a map $G: g(E) \rightarrow C l(g(E))$ by $G(g x)=T x$.

For $x, y \in E, T x=T y$ whenever $g x=g y$, i.e., $G(g x)=G(g y)$. Also, we have $T x \subset T(E) \subseteq$ $g(E)$. Thus, $G$ is a mapping. Now, for $g x, g y \in g(E)$ we have

$$
\frac{1}{2} d(g x, G(g x))<d(g x, g y) \quad \text { implies } \quad H(G(g x), G(g y))<\alpha M(g x, g y),
$$

where

$$
M(g x, g y)=\max \left\{d(g x, g y), d(g x, G(g x)), d(g y, G(g y)), \frac{1}{2}(d(g x, G(g y))+d(g y, G(g x)))\right\} .
$$


By Theorem 2.1, $G$ has a fixed point in $g(E)$, that is, there exists $g v \in g(E)$ such that $g v \in$ $G(g v)=T v$. Further, if $Y=X$, we have $g v \in T v$. Thus, $g g v \in g T v \subset T g v \subset T T v$. Now, we want to show that $g v=g g v$. Suppose $g v \neq g g v$, then we have

$$
\frac{1}{2} d(g v, T v)=0<d(g v, g g v), \quad \text { which implies } \quad H(T v, T g v)<\alpha M(g v, g g v),
$$

where

$$
M(g v, g g v)=\max \left\{d(g v, g g v), d(g v, T v), d(g g v, \operatorname{Tg} v), \frac{1}{2}(d(g v, \operatorname{Tg} v)+d(g g v, T v))\right\} .
$$

Since $g v \in T v, g g v \in T g v$ and $\frac{1}{2}(d(g v, T g v)+d(g g v, T v)) \leq H(T v, T g v)$, we obtain

$$
M(g v, g g v) \leq \max \{d(g v, g g v), H(T v, T g v)\} .
$$

Hence,

$$
H(T v, T g v)<\alpha \max \{d(g v, g g v), H(T v, T g v)\} \leq \alpha d(g v, g g v) .
$$

We have $d(g v, g g v) \leq d(g v, w)+d(w, g g v)$ for each $w \in T g v$. Since $\operatorname{Tg} v$ is a closed set, then

$$
d(g v, g g v) \leq d(g v, \operatorname{Tg} v)+d(g g v, \operatorname{Tg} v)
$$

but $g g v \in T g v$. Thus, $d(g v, g g v) \leq d(g v, T g v)$. Since $g v \in T v$, we have

$$
d(g v, \operatorname{Tg} v)<H(\operatorname{Tv}, \operatorname{Tg} v)<\alpha d(g v, \operatorname{Tg} v)
$$

which is a contradiction. Therefore, $g v=g g v \in T g v$.

Corollary 3.2 Let $(X, d)$ be a metric space, $Y$ be a nonempty set, $f, g: Y \rightarrow X$ such that $f(Y) \subseteq g(Y)$ and $g(Y)$ is a compact subspace of $X$. Assume that for $x, y \in Y$ there exists $\alpha \in\left[0, \frac{1}{2}\right)$ such that

$$
\frac{1}{2} d(g x, f x)<d(g x, g y) \quad \text { implies } \quad d(f x, f y)<\alpha M(g x, g y),
$$

where

$$
M(g x, g y)=\max \left\{d(g x, g y), d(g x, f x), d(g y, f y), \frac{1}{2}(d(g x, f y)+d(g y, f x))\right\}
$$

and $g x=$ gy implies $f x=f y$. Then $f$ and $g$ have a unique coincidence point. If $Y=X$, then $f$, $g$ have a unique common fixed point provided that $f$ and $g$ commute at $v$.

Proof The proof of this corollary follows from Theorem 3.1 by taking $T: Y \rightarrow X$. We need to prove that $v$ is a unique coincidence point of $f$ and $g$. Suppose, to the contrary, that there exists $z \in X$ such that $z \neq v$ and $f z=g z$. Then the inequalities $d(g v, g z)>0$ and $0=\frac{1}{2} d(g v, f v)<d(g v, g z)$ are satisfied. Thus, we have $d(f v, f z)<\alpha M(g v, g z)$, where

$$
M(g v, g z)=\max \left\{d(g v, g z), d(g v, f v), d(g z, f z), \frac{1}{2}(d(g v, f z)+d(g z, f v))\right\},
$$


i.e.,

$$
M(g v, g z)=\max \{d(g v, g z), 0\} .
$$

Therefore, $d(f v, f z)<\alpha d(g v, g z)$, that is, $d(g v, g z)<\alpha d(g v, g z)$, this is impossible due to $\alpha<\frac{1}{2}$. Hence, $v$ is the unique coincidence point. Moreover, if $Y=X$, we have $f g v=g f v$ but $f v=g v$. Thus, $f g v=g g v$, i.e., $g v$ is a coincidence point. Because of the uniqueness of the coincidence point, we obtain that $g v=v$. Then $v=g v=f v$.

\section{An application}

Many authors have studied the existence and uniqueness of solutions of functional equations and system of functional equations in dynamic programming by using diverse fixed point theorems (cf. [14-16]).

Throughout this section, we suppose that $U$ and $V$ are Banach spaces, $W \subseteq U$ is the state space, $D \subseteq V$ is the decision space and $\mathbb{R}$ is the field of real numbers. Let $B(W)$ denote the set of all the bounded real-valued functions on $W$. For an arbitrary $h \in W$, define $\|h\|=\sup _{x \in W}|h(x)|$. Then $(B(W),\|\cdot\|)$ is a Banach space.

The return functions $S, T: W \rightarrow \mathbb{R}$ of the continuous decision process are defined by the functional equations

$$
S=\sup _{y \in D}\{q(x, y)+G(x, y, S(\tau(x, y)))\}, \quad x \in W
$$

and

$$
T=\sup _{y \in D}\left\{q^{\prime}(x, y)+F(x, y, T(\tau(x, y)))\right\}, \quad x \in W,
$$

where $x$ and $y$ represent the state and decision vectors, respectively, $\tau: W \times D \rightarrow W$ represents the transformation of the process. Moreover, $q, q^{\prime}: W \times D \rightarrow \mathbb{R}$ and $G, F$ : $W \times D \times \mathbb{R} \rightarrow \mathbb{R}$ are bounded functions.

In this article, we prove the existence and uniqueness of the common solution of functional equations (4.1) and (4.2) arising in dynamic programming, using Corollary 3.2.

Let the maps $f$ and $g$ be defined by

$$
\begin{array}{ll}
f h(x)=\sup _{y \in D}\{q(x, y)+G(x, y, h(\tau(x, y)))\}, & x \in W, h \in B(W), \\
g h(x)=\sup _{y \in D}\left\{q^{\prime}(x, y)+F(x, y, h(\tau(x, y)))\right\}, & x \in W, h \in B(W) .
\end{array}
$$

Suppose that the following conditions hold.

(Q1) For any $h \in B(W)$, there exists $k \in B(W)$ such that

$$
f h(x)=g k(x), \quad x \in W .
$$

(Q2) There exists $h \in B(W)$ such that

$$
f h(x)=g h(x) \text { implies } \quad g f h(x)=f g h(x) .
$$


Theorem 4.1 Suppose that conditions (Q1) and (Q2) are satisfied and $g(B(W))$ is a closed and bounded subspace of $B(W)$. Assume that there exists $\alpha \in\left[0, \frac{1}{2}\right)$ such that for every $(x, y) \in W \times D, h, k \in B(W)$ and $t \in W$, the inequality

$$
\frac{1}{2}|f h(t)-g h(t)|<|g h(t)-g k(t)|
$$

implies

$$
|G(x, y, h(t))-G(x, y, k(t))|<\alpha M(g h, g k),
$$

where

$$
\begin{aligned}
M(g h, g k)= & \max \{|g h(t)-g k(t)|,|g h(t)-f h(t)|,|g k(t)-f k(t)|, \\
& \left.\frac{|g h(t)-f k(t)|+|g k(t)-f h(t)|}{2}\right\},
\end{aligned}
$$

then functional equations (4.1) and (4.2) have a unique common bounded solution in W.

Proof Note that, due to $q, q^{\prime}, G$ and $F$ are bounded, $f$ and $g$ are self-maps of $B(W)$. $(B(W), d)$ is a complete metric space, where $d$ is the metric defined by the supremum norm on $B(W)$. Since $g(B(W))$ is a closed and bounded subspace of $B(W)$, then $g(B(W))$ is complete and bounded. That is, $g(B(W))$ is compact. Conditions (Q1) and (Q2) imply that $f(B(W)) \subseteq g(B(W))$ and $f$ and $g$ commute at their coincidence points.

Let $\varepsilon$ be an arbitrary positive real number, and $h_{1}, h_{2} \in B(W)$. For $x \in W$, choose $y_{1}, y_{2} \in$ $D$ such that

$$
f h_{1}(x)<q\left(x, y_{1}\right)+G\left(x, y_{1}, h_{1}\left(\tau_{1}\right)\right)+\varepsilon
$$

and

$$
f h_{2}(x)<q\left(x, y_{2}\right)+G\left(x, y_{2}, h_{2}\left(\tau_{2}\right)\right)+\varepsilon,
$$

where $\tau_{1}=\tau\left(x, y_{1}\right)$ and $\tau_{2}=\tau\left(x, y_{2}\right)$.

Furthermore, from the definition of $f$, we have

$$
f h_{1}(x) \geq q\left(x, y_{2}\right)+G\left(x, y_{2}, h_{1}\left(\tau_{2}\right)\right)
$$

and

$$
f h_{2}(x) \geq q\left(x, y_{1}\right)+G\left(x, y_{1}, h_{2}\left(\tau_{1}\right)\right)
$$

If we suppose that the following inequality holds

$$
\frac{1}{2}\left|f h_{1}(x)-g h_{1}(x)\right|<\left|g h_{1}(x)-g h_{2}(x)\right|
$$

then

$$
\left|G\left(x, y_{1}, h_{1}\left(\tau_{1}\right)\right)-G\left(x, y_{1}, h_{2}\left(\tau_{1}\right)\right)\right|<\alpha M\left(g h_{1}, g h_{2}\right),
$$


where

$$
\begin{aligned}
M\left(g h_{1}, g h_{2}\right)= & \max \left\{\left|g h_{1}(x)-g h_{2}(x)\right|,\left|g h_{1}(x)-f h_{1}(x)\right|,\left|g h_{2}(x)-f h_{2}(x)\right|,\right. \\
& \left.\frac{\left|g h_{1}(x)-f h_{2}(x)\right|+\left|g h_{2}(x)-f h_{1}(x)\right|}{2}\right\} .
\end{aligned}
$$

From (4.3), (4.6) and (4.7), we obtain that

$$
\begin{aligned}
f h_{1}(x)-f h_{2}(x) & <G\left(x, y_{1}, h_{1}\left(\tau_{1}\right)\right)-G\left(x, y_{1}, h_{2}\left(\tau_{1}\right)\right)+\varepsilon \\
& \leq\left|G\left(x, y_{1}, h_{1}\left(\tau_{1}\right)\right)-G\left(x, y_{1}, h_{2}\left(\tau_{1}\right)\right)\right|+\varepsilon \\
& <\alpha M\left(g h_{1}, g h_{2}\right)+\varepsilon .
\end{aligned}
$$

Similarly, (4.4), (4.5) and (4.7) imply

$$
\begin{aligned}
f h_{2}(x)-f h_{1}(x) & <G\left(x, y_{1}, h_{2}\left(\tau_{1}\right)\right)-G\left(x, y_{1}, h_{1}\left(\tau_{1}\right)\right)+\varepsilon \\
& \leq\left|G\left(x, y_{1}, h_{1}\left(\tau_{1}\right)\right)-G\left(x, y_{1}, h_{2}\left(\tau_{1}\right)\right)\right|+\varepsilon \\
& <\alpha M\left(g h_{1}, g h_{2}\right)+\varepsilon .
\end{aligned}
$$

Thus, from (4.8) and (4.9) we have

$$
\left|f h_{1}(x)-f h_{2}(x)\right|<\alpha M\left(g h_{1}, g h_{2}\right)+\varepsilon
$$

Since $\varepsilon$ is arbitrary, therefore for any $x \in W$, we have

$$
\frac{1}{2}\left|f h_{1}(x)-g h_{1}(x)\right|<\left|g h_{1}(x)-g h_{2}(x)\right| \text { implies }\left|f h_{1}(x)-f h_{2}(x)\right|<\alpha M\left(g h_{1}, g h_{2}\right),
$$

where

$$
\begin{aligned}
M\left(g h_{1}, g h_{2}\right)= & \max \left\{\left|g h_{1}(x)-g h_{2}(x)\right|,\left|g h_{1}(x)-f h_{1}(x)\right|,\left|g h_{2}(x)-f h_{2}(x)\right|,\right. \\
& \left.\frac{\left|g h_{1}(x)-f h_{2}(x)\right|+\left|g h_{2}(x)-f h_{1}(x)\right|}{2}\right\} .
\end{aligned}
$$

Therefore, by Corollary 3.2, $f$ and $g$ have a unique common fixed point, and hence functional equations (4.1) and (4.2) have a unique bounded common solution.

Remark 4.2 Fukhar-ud-din et al. [17] have established fixed point results on a noncompact domain in uniformly convex metric spaces for a single-valued map satisfying a contractive condition closely related to the Suzuki condition employed in Theorem 1.2. It will be interesting to extend the results of this paper in this general setup ( $c f$. Open problem 1 on p.4759 in [17]). 
Authors' contributions

All authors contributed equally and significantly in writing this paper. All authors read and approved the final version.

\section{Author details}

'School of Mathematical Sciences, Faculty of Science and Technology, Universiti Kebangsaan Malaysia, UKM, Bangi, Selangor Darul Ehsan 43600, Malaysia. ²Department of Mathematics, King Abdulaziz University, P.O. Box 138381, Jeddah, 21323, Saudi Arabia.

\section{Acknowledgements}

The authors would like to acknowledge the financial support received from Universiti Kebangsaan Malaysia under the research grant UKM-DIP-2012-31.

Received: 22 July 2013 Accepted: 4 December 2013 Published: 02 Jan 2014

\section{References}

1. Von Neuman, J: Über ein ökonomisches Gleichungssystem und eine Verallgemeinerung des Brouwerschen Fixpunktsatzes. Ergeb. Math. Kolloq. 8, 73-83 (1937)

2. Nadler, SB Jr.: Multi-valued contraction mappings. Pac. J. Math. 30, 475-488 (1969)

3. Nadler, SB Jr.: Hyperspaces of Sets: A Text with Research Questions. Monographs and Textbooks in Pure and Applied Mathematics, vol. 49. Dekker, New York (1978)

4. Doric, D, Lazovic, R: Some Suzuki-type fixed point theorems for generalized multivalued mappings and applications. Fixed Point Theory Appl. 2011, Article ID 40 (2011)

5. Ciric, LB: Fixed points for generalized multi-valued contractions. Mat. Vesn. 9(24), 265-272 (1972)

6. Kikkawa, M, Suzuki, T: Three fixed point theorems for generalized contractions with constants in complete metric spaces. Nonlinear Anal. 69, 2942-2949 (2008)

7. Suzuki, T: A new type of fixed point theorem in metric spaces. Nonlinear Anal. 71, 5313-5317 (2009)

8. Edelstein, M: On fixed and periodic points under contractive mappings. J. Lond. Math. Soc. 37, 74-79 (1962)

9. Abtahi, M: A fixed point theorem for contractive mappings that characterizes metric completeness. J. Math. Anal. Appl. arXiv:1207.6207v1 (2012)

10. Bari, CD, Vetro, P: Common fixed points for self mappings on compact metric spaces. Appl. Math. Comput. 219, 6804-6808 (2013)

11. Doric, D, Kadelburg, Z, Radenović, S: Edelstein-Suzuki-type fixed point results in metric and abstract metric spaces. Nonlinear Anal. 75, 1927-1932 (2012)

12. Singh, SL, Mishra, SN: Remarks on recent fixed point theorems. Fixed Point Theory Appl. 2010, Article ID 452905 (2010)

13. Haghi, RH, Rezapour, S, Shahzad, N: Some fixed point generalizations are not real generalizations. Nonlinear Anal. 74, 1799-1803 (2011)

14. Khan, AR: Common fixed point and solution of nonlinear functional equations. Fixed Point Theory Appl. 2013, 290 (2013)

15. Popescu, O: A new type of contractive multivalued operators. Bull. Sci. Math. 137, 30-44 (2013)

16. Singh, SL, Mishra, SN: Fixed point theorems for single-valued and multi-valued maps. Nonlinear Anal. 74, 2243-2248 (2011)

17. Fukhar-ud-din, H, Khan, AR, Akhtar, Z: Fixed point results for a generalized nonexpansive map in uniformly convex metric spaces. Nonlinear Anal. 75, 4747-4760 (2012)

10.1186/1029-242X-2014-7

Cite this article as: Shaddad et al.: Common fixed point results of Ciric-Suzuki-type inequality for multivalued maps in compact metric spaces. Journal of Inequalities and Applications 2014, 2014:7

\section{Submit your manuscript to a SpringerOpen ${ }^{\ominus}$ journal and benefit from:}

- Convenient online submission

- Rigorous peer review

- Immediate publication on acceptance

Open access: articles freely available online

- High visibility within the field

- Retaining the copyright to your article 